\title{
Virtual Reality for Early Education: A Study
}

\author{
Adeola Fabola and Alan Miller \\ School of Computer Science \\ Universitiy of St Andrews \\ United Kingdom \\ \{aef6, alan.miller\}@st-andrews.ac.uk \\ http://www.cs.st-andrews.ac.uk/
}

\begin{abstract}
This paper investigates the use of Virtual Reality (VR) as a tool for cultural heritage learning, using St Andrews Cathedral as the subject matter. As part of a module focused on local history, first year secondary school pupils in a school in the town of St Andrews took part in virtual tours of the Cathedral as it stood in the $14^{\text {th }}$ Century using the Samsung Gear VR, Google Cardboard, Oculus Rift, computer screen and Xbox controller, and answered questions aimed to elicit their experiences with the various systems. The system design and implementation is presented and the findings, observations and lessons learnt from the study are discussed.
\end{abstract}

Keywords: Virtual Reality, Google Cardboard, Samsung Gear VR, cultural heritage

\section{Introduction: Heritage Interpretation in St Andrews}

St Andrews Cathedral is one of the most iconic monuments in Scottish history, as it was the center of religious activities and domicile of eminent figures in its heyday. Owing to its importance, the Open Virtual Worlds Group at the University of St Andrews embarked on a reconstruction of the Cathedral as it stood in 1318, and this reconstruction has been a valuable resource in exploring and teaching cultural heritage through deploying it in schools, museum installations and over the Internet [1].

St Andrews Cathedral reconstruction has served as the basis upon which other resources have been built. Pertinent to this paper, is the development of a mobile tour of St Andrews Cathedral which works with the Google Cardboard [2] and the Samsung Gear VR. These systems have been deployed in a school in the town of St Andrews in Scotland and used as the basis for learning local history as part of a module.

A study carried out to investigate the efficacy of such technology for learning history is discussed in this paper. Section 1.1 summarises some work done on using virtual reality for learning and hones in on cultural heritage, section 2 discusses the study and provides an overview of the system implementation and features, section 3 discusses the data analyses and results, and section 4 provides a conclusion and summary of lessons learnt. 

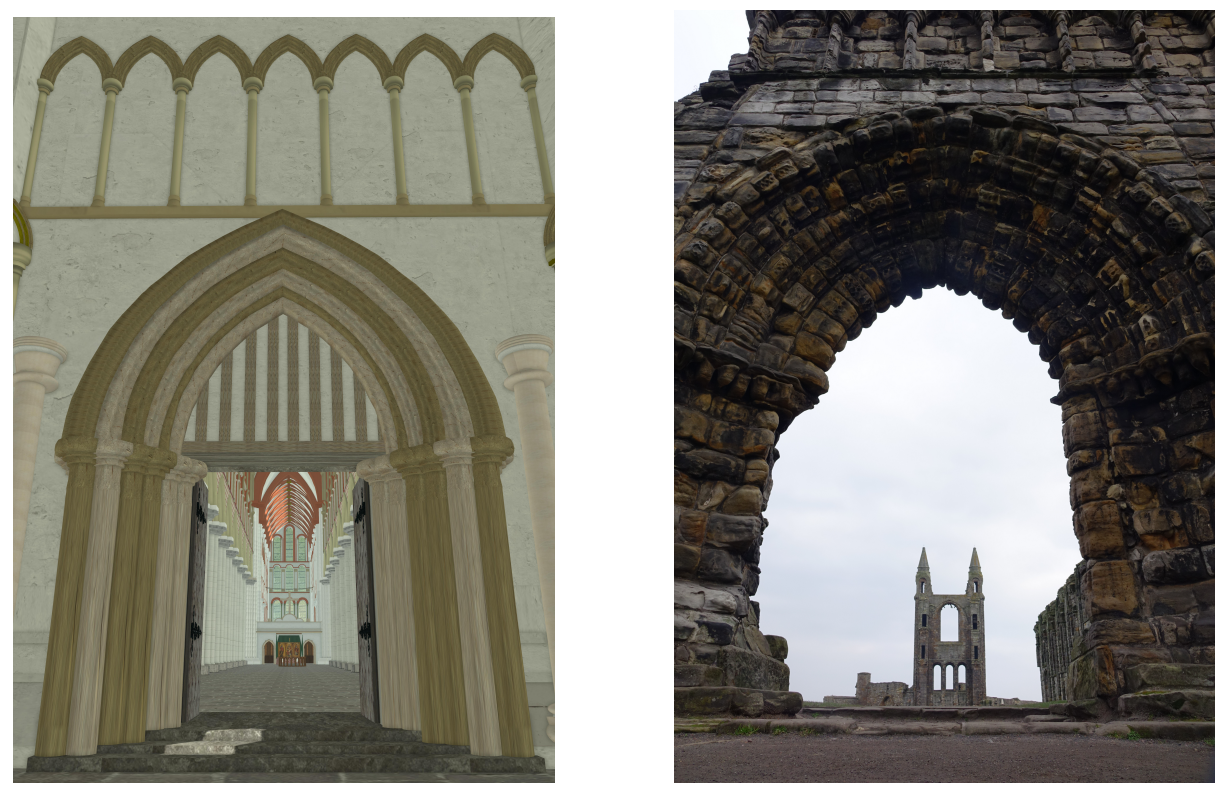

Fig. 1: St Andrews Cathedral West Door: 1318 (left) and today (right)

\subsection{Related Work: Virtual and Augmented Reality for Learning}

It is important to define what we mean by the concepts of Virtual Reality (VR) and Augmented Reality (AR) and situate them in the context of this work. VR refers to systems characterised by environments which are comprised solely of artificial elements courtesy of computer-generated imagery, and represent one extreme in the Reality-Virtuality Continuum proposed in [3], with the other extreme representing the real environment which is the world governed by the laws of physics e.g. gravity, time, space and so on. Between the Virtual Environment and Real Environment exists the concept of Mixed Reality (MR), which represents systems made up of real and virtual environments [3]. Augmented Reality (AR) refers to a class of Mixed Reality systems where a real environment is annotated with virtual (or synthetic) elements, in order to add information and context, or otherwise improve the real environment. This work focuses on VR because it facilitates the distinct exploration of two realities (the virtual and the real) in parallel, whereas AR merges both realities into one.

Virtual reality environments are equipped with certain features - such as the ability to facilitate shared experiences, encourage natural user interaction and enable unique learning experiences to meet individuals' needs - that make them suitable for learning [4]. Three challenges to the adoption of virtual reality for education are identified in [4]: cost, usability and fears of the technology. These challenges posed significant drawbacks to the adoption of virtual reality in the early 90's (and before). However, reductions in cost, and increases in the power 
of microprocessors and improved user interfaces have led to new paradigms that enhance usability and accessibility of VR systems.

The growing popularity of virtual reality for learning is investigated and confirmed by [5], which present a review of empirical studies conducted on the application of virtual reality for learning from 1999 to 2009. It is evident from this work that in the last decade, virtual reality has been leveraged for learning in a broad range of disciplines from the sciences to the social sciences, and that virtual reality is appropriate for teaching and learning, as well as for training and entertainment purposes [6]. The use of virtual reality as a tool for learning has been investigated by several scholars. Studies conducted by [7] suggest that virtual reality technologies can serve not only as an invaluable resource but also as an effective tool for learning and teaching. A virtual reality system for teaching routing algorithms by leveraging the graphical capabilities of an open source virtual reality platform to visualise network packets on a routing island is introduced in [8], [9] presents a case study for teaching a university module using a shared, 3D virtual environment, and [1] discusses the deployment of the 3D model in several scenarios such as museum exhibitions, science centres, schools and festivals.

Collaborative learning systems have also been developed using virtual reality technology. [10] recognises the importance of social connectedness amongst learners and thus facilitates collaboration through features such as dedicated meeting spaces (such as coffee areas) that mimic real-world environments. The authors conducted an initial study to ascertain the acceptance of the system as a collaborative learning tool and the results suggest that with a few exceptions, users are receptive to the use of such systems for learning. On-site exploration of heritage sites using mobile technology has also been largely discussed in the public domain. A Virtual Time Window (VTW) which facilitates on-site exploration of heritage sites using an open source virtual reality server and a mobile (tablet) client viewer is introduced in [11], [12] introduces a location-aware, Augmented Reality (AR) mobile application for exploration of a historical street, [13] discusses the use of a "serious game" for heritage learning during museum visits, and the use of Google Cardboard [18] to facilitate an on-site comparison of the past and present states of St Andrews Cathedral, armed with location-awareness and audio narratives was discussed in [2].

The concept of experiential learning has also been facilitated by virtual reality systems, for instance, [15] leverage immersive, game-based virtual reality systems to simulate the experience of archaeological excavations so as to foster a better understanding of the process, and the application of game-like methodologies to the learning process in this system is presented in [14]. The application of virtual reality for learning has also been investigated from a pedagogical standpoint. [16] investigate learners' attitudes towards virtual reality learning environments. The authors conclude that appropriate teaching practices should be taken into consideration in the development of virtual reality learning environments, and suggest that the effectiveness of using virtual reality for learning should be further explored. This is the motivation for this study. 

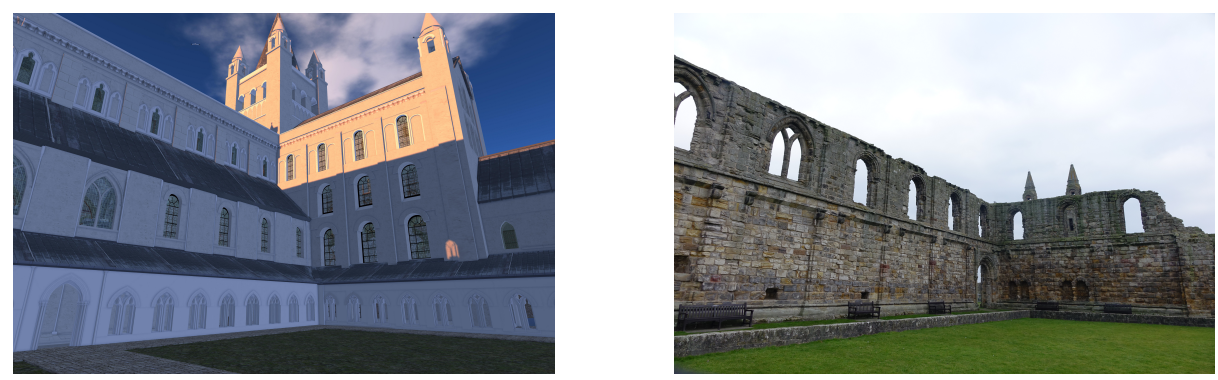

Fig. 2: St Andrews Cathedral Cloister: 1318 (left) and today (right)

\section{Study: A Comparative Analysis of VR Systems}

The systems used for the study were built using several tools and frameworks. 3D modelling software was used in the reconstruction of St Andrews Cathedral, while game engines and open-source libraries were used to deploy it to mobile platforms and to interconnect the components. Open Simulator (OpenSim), an open-source virtual environment was used to develop the 3D model of St Andrews Cathedral [1]. For the game engines, a few alternatives were explored. Unity3D (versions 4 and 5) [20] and Unreal Engine 4 [21] were chosen and both engines were used to develop two versions of the mobile system. The system built using the Unity 3D (version 4) was ultimately chosen for the study owing to better performance on the mobile platforms.

The system architecture employed for the mobile platforms is shown in Fig. 3. The system takes advantage of inbuilt features of commodity smartphones. The multimedia resources (images, audio and video) are stored on the device's storage, the gyroscope is used for head-tracking to provide a $360^{\circ}$ view of the environment, the GPS is used for location-awareness and the screen provides touch input into and visual output from the system. Four system set-ups were implemented: Samsung Gear VR (SG, Fig. 4b), Google Cardboard (GC, Fig. 4a), Oculus Rift and Xbox controller (OX) as well as screen, mouse and keyboard with Xbox controller (SX).

The Samsung Gear VR and Google Cardboard facilitated discrete exploration of the Cathedral from distinct viewpoints. These systems featured ten locations, obtained from the 3D reconstruction of St Andrews Cathedral (see [1]), linked together to form a trail. The locations were made out of spherical panoramas (Fig. 5a and Fig. 5a), as described in [2]. Audio narratives were associated with each location to serve as tour guides and additional content for the user. A significant difference between the Samsung Gear VR and the Google Cardboard systems was the navigation technique. i.e. the mechanism for moving from one location to another. On the Google Cardboard, navigation was hands-free by means of hotspots which are triggered when they overlap with a central crosshair for a few seconds. On the Samsung Gear VR, navigation took the form of a hands-on approach facilitated by a touch-pad on the side of the headset. 


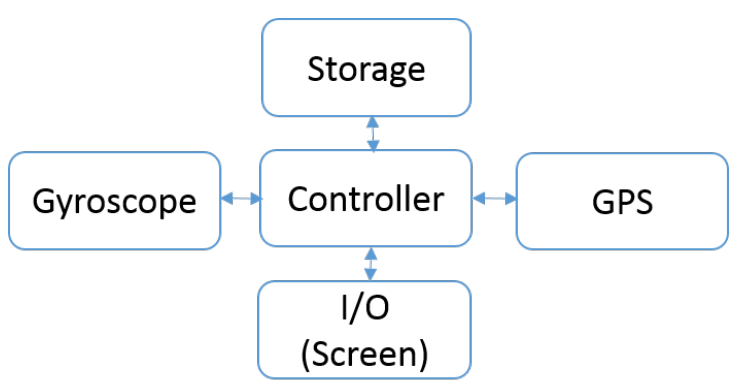

Fig. 3: System architecture

In contrast, the use of the Xbox controller with the Oculus Rift and the computer screen facilitated continuous exploration of the Cathedral. With these systems, the subjects had the freedom to explore the 3D model with an avatar that can walk, run and fly around the 3D space. Subjects had the option to see the Cathedral from different perspectives and view points, as they could toggle between a first-person view and a third-person view. It is important to note that although the mobile systems restricted subjects' exploration to distinct viewpoints, their mobile nature allowed for on-site exploration, such that subjects could walk around the (remains of the) actual site of the Cathedral while viewing the virtual reconstruction from equivalent vantage points. This would not have been possible with the screen and/or Oculus Rift tethered to a computer, as the size and power requirements confine its usage to specific (usually indoor) locations. The distinction between the different set-ups made for an interesting comparison of the benefits and limitations of each, as discussed in the sections that follow.

\subsection{Method: Data Collection and Feedback}

The four systems were set up in the library of a local school for a week, and cohorts of first year pupils were invited to explore St Andrews Cathedral using the systems. Each cohort comprised of up to 30 pupils and they were further divided into groups of 5 , each of which took turns in exploring the four systems. Data collection was by means of questionnaires and observation, with both quantitative and qualitative data collected.

"Background Information" for each pupil was recorded. Each pupil then had up to 10 minutes to explore the cathedral using each system, after which they filled the "Experience" section of the questionnaire shown in Table 2 on a fivepoint Likert scale describing their experiences enabling qualitative analysis. The averages of subjects' responses to each questionnaire item for each set-up was calculated and charted in order to observe a holistic view of the responses. For each questionnaire item, a one-way Analysis of Variance (ANOVA) was conducted across the four set-ups to determine whether there was a statistically-significant difference in the means. A statistically-significant difference would suggest that the subjects felt differently about the set-ups for a particular questionnaire item, 

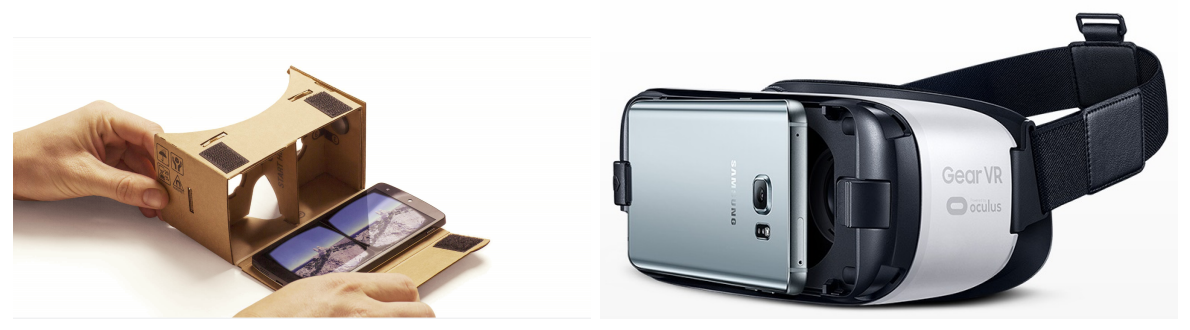

Fig. 4: Mobile VR Headsets: Google Cardboard [18] \& Samsung Gear VR [19]

while the absence of a statistically-significant difference would suggest otherwise. For cases where a statistically-significant difference was observed, a t-Test (TwoSample assuming unequal variances) was conducted with set-up pairs (i.e. SG and GC, SG and SX, GC and SX, and so on) to determine which pairs have means with statistically-significant differences. In order to determine the impact that the systems had on the subjects, correlation was conducted between the subjects' responses to some of the User Experience Items and Background Information; the rationale for this is explained further in sections 2.2 and 3.

The questionnaire also provided spaces for the pupils to provide three words describing their experience and to suggest what aspects of the experience could be improved on. In addition to these, the pertinent actions and spoken words of participants as observed by the researchers were noted. These data were collected for qualitative analyses (see section 3). Specifically, the three words requested from each of the participants were used to form a tag cloud and a frequency analysis was conducted to observe the most occurring words in the sequence, which would provide a holistic representation of the user experience. Similarly, the pupils' responses to what aspects of the experiences could be improved on, in conjunction with their actions and spoken words while using the system, were analysed to observe recurring patterns. These are discussed in section 3 .

\subsection{Hypotheses: Statistical Tests and Inferences}

The following hypotheses were proposed for the study:

$\mathrm{H}_{0}$ : The headset-based set-up will stimulate more interest in learning history than the screen-based set-up. This will be verified by analysing the means of subjects' responses to Item 4 across the Samsung Gear VR, Google Cardboard (headset-based set-ups) and the Screen and Xbox Controller. A One-way Analysis of Variance (ANOVA) will be conducted to determine whether there are statistically-significant differences between the means of the set-ups and if there are, a t-Test (Two-Sample assuming unequal variances) on pairs of the means of SG and SX, and GC and SX will be conducted. A statistically-significant difference between the means would suggest a difference in the levels of stimulation provided by the set-ups and in such a case, a comparison of the means would reveal which set-ups stimulate more interest in the subjects. 

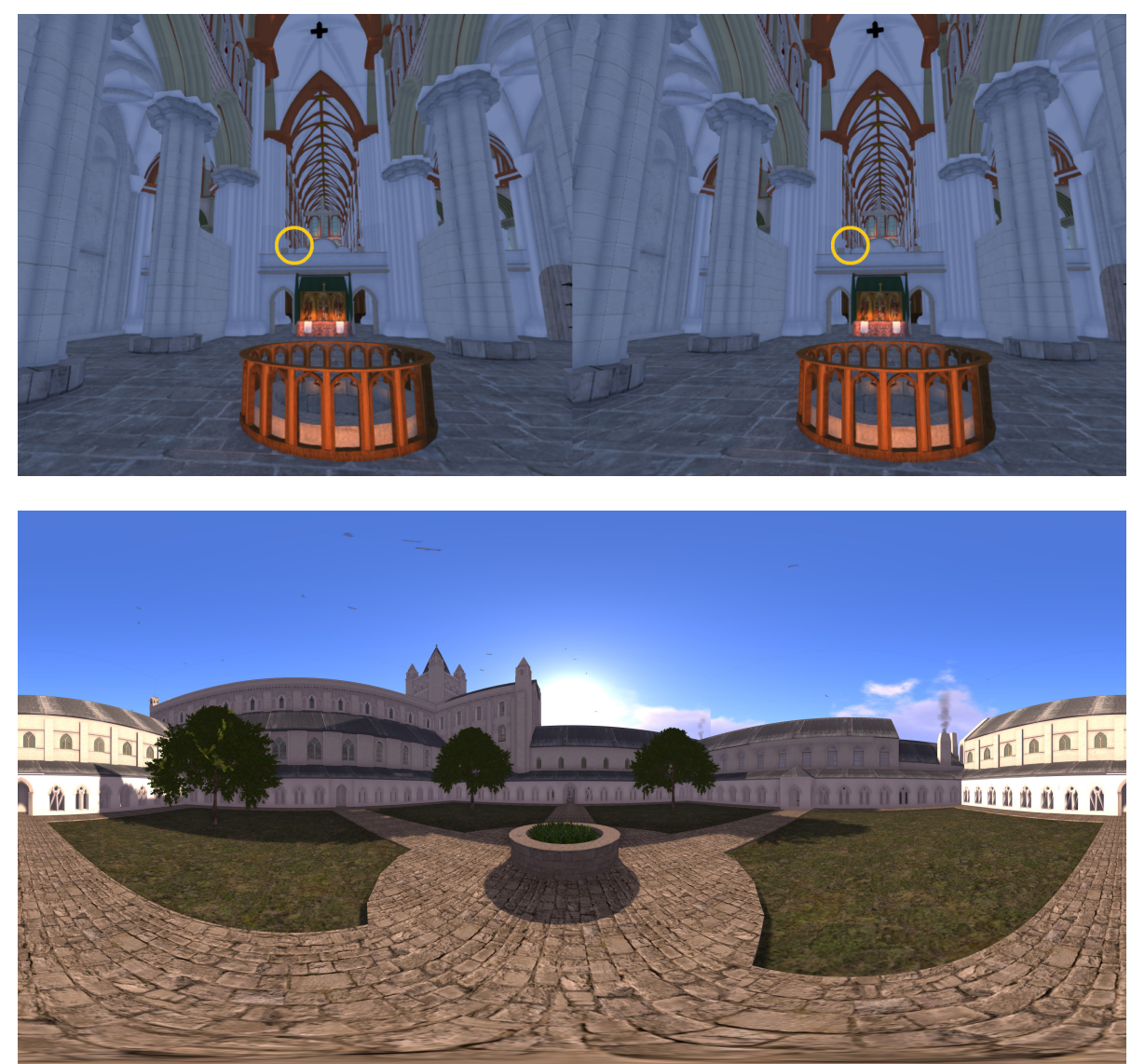

Fig. 5: Reconstruction of the Nave and projection of Cloister

$\mathrm{H}_{1}$ : The headset-based set-up will provide more immersion than the screenbased set-up. This will be verified by analysing the means of subjects' responses to Item 5 across the Samsung Gear VR, Google Cardboard (headset-based setups) and the Screen and Xbox Controller. A One-way ANOVA will be conducted to determine whether there are statistically-significant differences between the means of the set-ups and if there are, a t-Test (Two-Sample assuming unequal variances) on pairs of the means of SG and SX, and GC and SX will be conducted. A statistically-significant difference between the means would suggest a difference in the level of immersion provided by the set-ups and in such a case, a comparison of the means would reveal which set-ups provide more immersion than others.

$\mathrm{H}_{2}$ : Virtual Reality is easy to use regardless of prior experience. This will be verified by correlating subjects' previous experience with Virtual Reality (Background Item 4) with subjects' perceived ease of use of the system (Item 1). The absence of a strong positive correlation between Background Item 4 and Item 1 will suggest that there is no relationship between subjects' prior experience 
Background Information 1 - How good are your English skills?

Background Information 2 - How good are your IT skills?

Background Information 3 - How interested are you in History?

Background Information 4 - Do you have previous experience with Virtual Reality (VR)?

Background Information 5 - Do you have previous experience with VR Headsets?

Table 1: Subject Background Information (ranked on a three-point Likert scale)

Item 1 (Easy to use) - I think that this system is easy to use

Item 2 (Would recommend) - I would recommend this system for learning history

Item 3 (Changed perception) - This system has changed how I think about the Cathedral

Item 4 (Stimulated interest) - I am now more interested in learning about local history

Item 5 (Immersion) - I felt like I was there in the virtual environment

Table 2: User Experience Items (ranked on a five-point Likert scale)

with virtual reality and subjects' perceived ease of use. This, coupled with high mean scores for subjects' responses to Item 1 would suggest that virtual reality is easy to use regardless of prior experience.

\section{Analyses, Results and Discussions}

The data collected was analysed using a combination of qualitative and quantitative techniques. Qualitative techniques took the form of a word analysis on the subjects' feedback and observations of their actions while statistical techniques such as a One-way Analysis of Variance (ANOVA), t-tests and correlation were used to analyse the quantitative data.

Qualitative Data Analysis. A tag cloud was created to visualise the most common words used by the pupils to describe their experiences. A frequency analysis of the tag cloud (shown in Fig. 6) revealed that the top twelve (12) words were "fun", "interesting", "cool", "amazing", "good", "realistic", "awesome", "exciting", "educational", "real", "weird" and "different". Each word was placed in one of three categories depending on whether the experience described by the word was positive, negative or neutral. Negative words (such as weird, scary, uncontrollable) and neutral words (such as different, tricky) combined accounted for $\approx 6 \%$ of the total words while $\approx 94 \%$ of the words were positive. Table 3 shows the result of a frequency analysis carried out on the words the subjects used to describe their experience. The observations made during the study are described in the following section.

Observations. The shape, size and physical features of VR headsets can contribute to and/or inhibit user experience. For instance, the Samsung Gear VR is designed to shut off the display and audio when there is no proximity sensor reading i.e. when the headset is not being worn. As the study subjects were preteen children, the size of the Gear VR meant that it was too big for the heads 


\begin{tabular}{|l|l|l|l|l|}
\hline Position & Word & Frequency & Percentage & Category \\
\hline 1 & fun & 44 & 10.45 & Positive \\
\hline 2 & interesting & 38 & 9.03 & Positive \\
\hline 3 & cool & 33 & 7.84 & Positive \\
\hline 4 & amazing & 23 & 5.46 & Positive \\
\hline 5 & good & 17 & 4.04 & Positive \\
\hline 6 & realistic & 13 & 3.09 & Positive \\
\hline 7 & awesome & 10 & 2.38 & Positive \\
\hline 7 & exciting & 10 & 2.38 & Positive \\
\hline 9 & educational & 9 & 2.14 & Positive \\
\hline 10 & real & 8 & 1.90 & Positive \\
\hline 10 & weird & 8 & 1.90 & Negative \\
\hline 10 & different & 8 & 1.90 & Neutral \\
\hline
\end{tabular}

Table 3: Frequency analysis of subjects' descriptive words

of some of them (even after fastening the strap as tight as possible) and this caused the headset to lose contact with their faces and by extension lose contact with the proximity sensor, often resulting in a break in transmission.

Cues (such as eye level) in the virtual environment play a part in the user experience. It was observed that the eye levels in the virtual scenes did not align with the eye levels of the subjects. This is because the virtual scenes were taken at the eye level of an average male, which is higher than the eye level of the preteen subjects. Some of the subjects expressed perceived dissonance as a result of this, while others did not report any such symptoms. A subject also reported the strange feeling of being "there [in the virtual world] but not there", as they felt like they were in the virtual environment but knew that they were seated in a chair in the real world.

In addition to visual cues, audio cues can play a big role in the user experience. During the study a subject was observed trying to put the headset (Google Cardboard) close to their ear, and by implication taking the headset away from their face, perhaps to hear the audio narrative. This could suggest that the subject wished for louder audio and needed the narrative to bolster the experience. In attempting to improve the audible experience (by hearing louder audio), the subjects inadvertently broke the visual experience. This may not have been the case if the audio was loud enough for them, but as it wasn't, they were willing to sacrifice the visual experience for the audible one. This could suggest that the audio is just as (or perhaps more) important than the visuals in virtual reality.

It was observed that the subjects expressed themselves more when they used the device with other subjects present; and they were quieter when they used the device alone. Subjects were also observed asking each other where they were on the tour, as if to suggest that they wanted to be at the same places at the same time. This could affirm the case for social exploration of heritage sites [17].

The interaction paradigm plays a role in the user experience. A subject was observed trying to lean forward and backward to zoom in and out respectively. This suggests that the immersive experience provided by the system caused the 


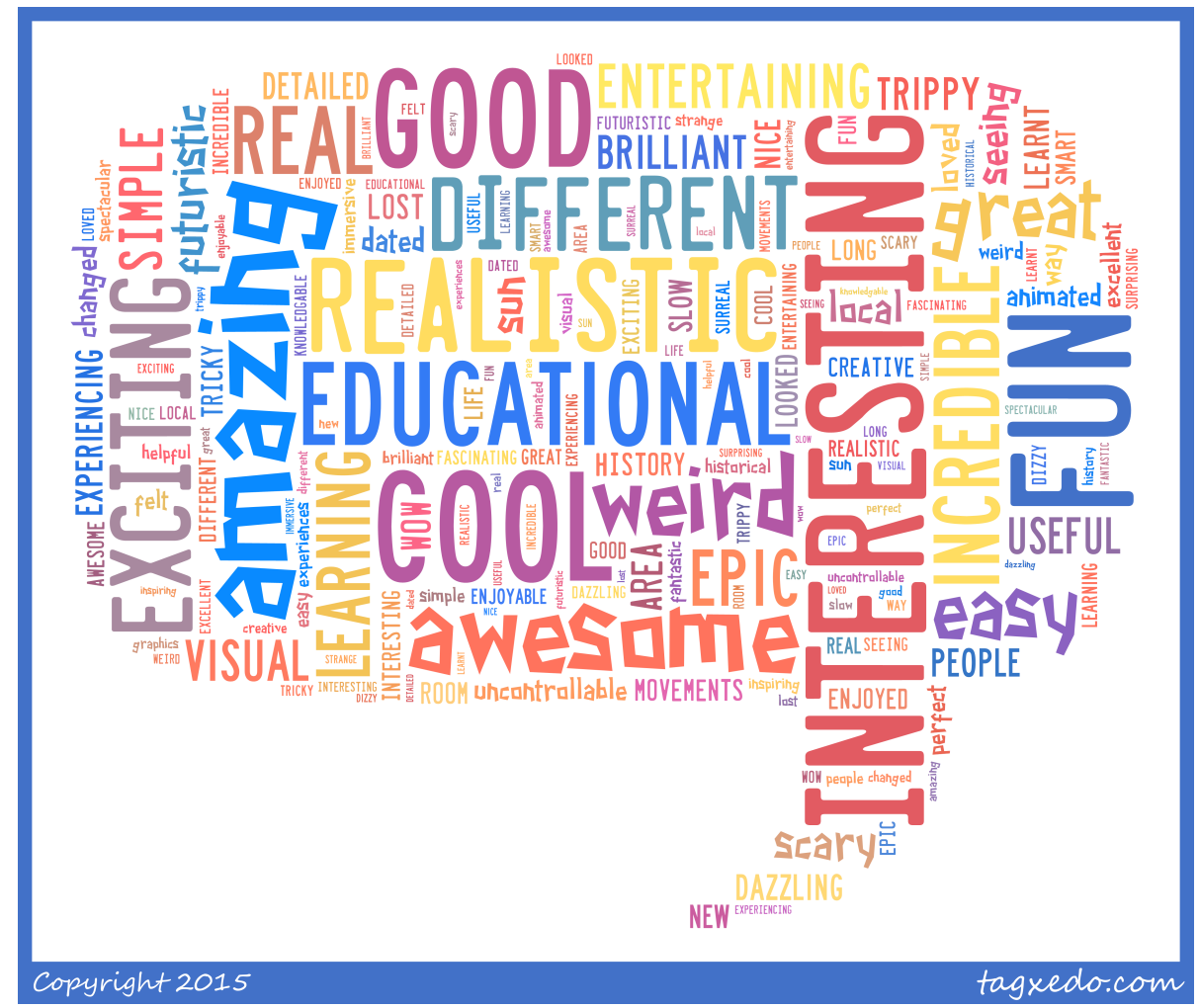

Fig. 6: Tag cloud visualising words participants used to describe their experiences

subject to assume that by leaning forward in real-life, their view in the virtual environment would become closer. For this reason, a "lean-to-zoom" feature (where a user leans forward or backward to zoom in or out respectively) could be valuable (and intuitive) in the use of virtual reality headset systems. Such a feature is currently being developed for use in a future release of the system with a view to investigate the level of usefulness and intuitiveness. Also, it was observed that swivel chairs facilitate better exploration of $360^{\circ}$ environments while seated, because they provide the ability to completely rotate around a fixed axis as compared to non-swivel chairs. This is not an issue while exploring $360^{\circ}$ environments while standing; however, standing poses an increased risk of injury (by falling or bumping into walls or objects for example).

Virtual reality can be a valuable tool for learning (as proven by $\mathrm{H}_{0}$ in section 3 ), but this may not always be the case. It was observed that for some subjects, the fun factor of the system detracted from the learning objective. This could suggest that there may be some conditions - configurations, content, scenarios and so on - under which a virtual reality system could be an effective learning tool, and others under which it would not be ideal for learning. This warrants further investigation. 


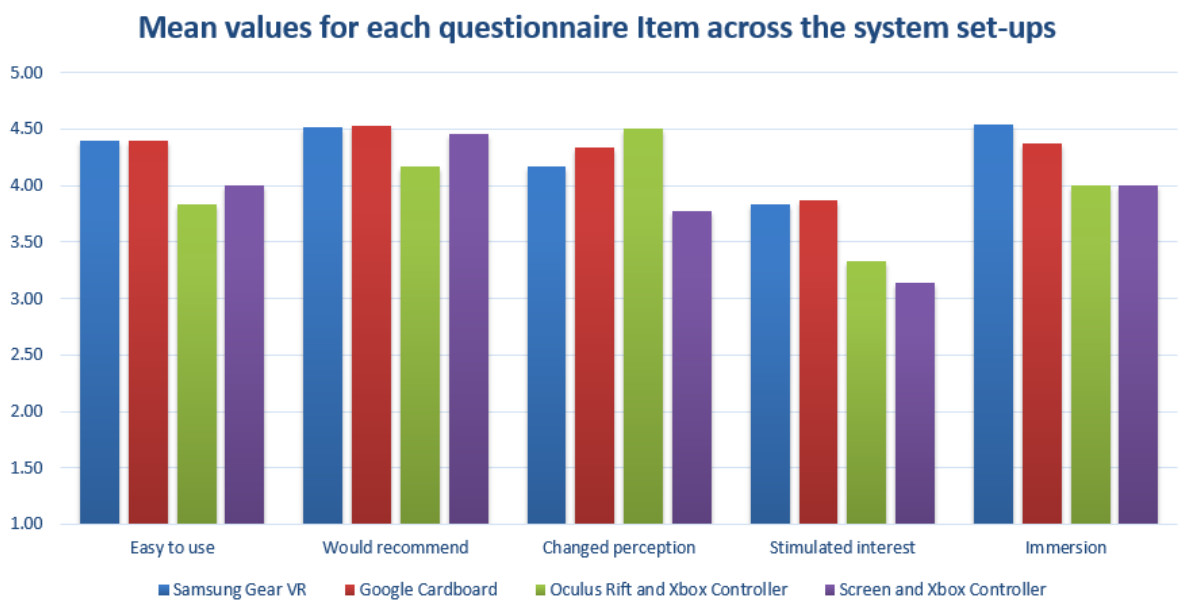

Fig. 7: Mean values for each questionnaire Item across the system set-ups

User inclinations and preferences can determine the nature of the experience. For instance, it was observed a few subjects refrained from properly fastening the headset strap over their heads. The experiences of said subjects were limited, as their hands were occupied with holding up the device on their faces, and this occasionally resulted in shaky movements that led to a loss of contact with the proximity sensor which resulted in a break in transmission. This suggests that users' inclinations (such as vanity, timidity, fear and so on) can get in the way of effective device usage and consequently inhibit the experience.

Some statements uttered by the subjects while interacting with the system are highlighted below: "Like a big, massive cinema screen" "Nice shadows" referring to the realistic nature of the digital model viewed in the display "I hear people but I don't see them" "I like this world better than the world we are in" "It's weird and really sickening" "Don't look at this if you're scared of heights" - referring to scenes with significantly raised eye levels "It's a shame you can't walk about yourself" "Ooh...scary" - said with excitement "This virtual life is really confusing"

Quantitative Data Analysis. The mean scores and a one-way ANOVA test was carried out on the data. A snapshot of the data collected is shown in Fig. 7. The feedback to each questionnaire item was ranked on a five-point Likert scale, where 1 (strongly disagree) and 2 (probably disagree) represent negative feedback, 3 (neither agree nor disagree) represents neutral feedback, and 4 (probably agree) and 5 (strongly agree) represent positive feedback. As shown in Fig. 7, the means of all four set-ups for Item 1 are positive (i.e. above neutral or greater than 3). However, a One-way (ANOVA) of subjects' responses to Item 1 for the four set-ups revealed that there is no statistically-significant difference in the means. This suggests that subjects felt that the four virtual reality systems are easy to use, but no one set-up is significantly easier to use than others. 
As shown in Fig. 7, the means of all four set-ups for Item 2 are positive. However, a One-way ANOVA of subjects' responses to Item 2 revealed that there is no statistically-significant difference in the means. This suggests that the subjects would recommend virtual reality as a tool for learning history; this is in line with previous work [7][12][13] and for learning in general [8][9]. However, the lack of a statistically-significant difference in the means suggests that the subjects would not necessarily recommend one type of the four virtual reality set-ups over another for learning history.

As shown in Fig. 7, the means of all four set-ups for Item 3 are positive. However, a One-way ANOVA of subjects' responses to Item 3 revealed that there is no statistically-significant difference in the means. This suggests that the subjects' views about St Andrews Cathedral were changed by the virtual reality systems, but no one set-up influenced the subjects views of St Andrews Cathedral more than the others. This demonstrates the impact that virtual reality systems can have on users when deployed in a learning context.

For Item 4, it was observed that the means for all four set-ups are positive, which suggests that the subjects' interests in learning history were stimulated by the systems. A One-way ANOVA of subjects' responses revealed that there is a statistically-significant difference in the means, which suggests that some set-ups stimulated the subjects interests more than the others. Further tests to determine which means are different were conducted. A t-Test (Two-Sample assuming unequal variances) on pairs of the means revealed that there is a statisticallysignificant difference between the Samsung Gear VR and the Screen with Xbox Controller, and also between the Google Cardboard and the Screen with Xbox Controller. This, coupled with the higher mean responses for the Samsung Gear VR (3.83) and the Google Cardboard (3.88) as compared to the Screen with Xbox Controller (3.14), suggests that the Samsung Gear VR and the Google Cardboard individually stimulated the subjects' interests more than the Screen with the Xbox Controller. This confirms $\mathrm{H}_{0}$.

As shown in Fig. 7, the means of all four set-ups for Item 5 are positive, with the means for the Samsung Gear VR (4.54) and Google Cardboard (4.38) higher than the mean of the Screen with Xbox Controller (4.00). However, a One-way ANOVA of subjects' responses to Item 5 did not find a statistically-significant difference in the means. This suggests that the subjects felt immersed in the virtual environment, but it does not confirm that any one set-up provided more immersion than the others and thus does not confirm $\mathrm{H}_{1}$. This warrants further investigation.

A correlation of the subjects' previous experience with Virtual Reality (Background Item 4) with subjects' perceived ease of use of the system (responses to Item 1 for all the set-ups) revealed a weak, positive correlation of 0.01 . The weak correlation suggests that there is little relationship between prior experience with virtual reality and perceived ease of use of the system, hence the positive mean scores $(4.40,4.39,3.83$, and 4.00 on a 5 -point scale for SG, GC, OX and SX respectively) recorded by the subjects for ease of use suggests that the system is easy to use regardless of prior experience and thus proves $\mathrm{H}_{2}$. 


\section{Conclusion}

The efficacy of virtual reality as a tool for learning history has been demonstrated in this work. Virtual reality has the potential to stimulate interest in a subject matter and present information in engaging and interactive ways. The findings of this study suggest that several factors can contribute to (or otherwise inhibit) the user experience. Visual and audio cues can enhance the experience, and other factors such as the device shape, size and interaction mechanism determine the nature of the user experience. Overall, given the appropriate conditions, virtual reality systems can be a suitable tool for learning history in early education. This study investigates three hypotheses $-\mathrm{H}_{0}, \mathrm{H}_{1}$ and $\mathrm{H}_{2}$. The method used was a combination of quantitative and qualitative techniques (see section 2.1) and the results (see section 3) confirmed $\mathrm{H}_{0}$, that headset-based virtual reality systems stimulate young pupils' interest in learning history more than screen-based virtual reality systems, and $\mathrm{H}_{2}$, that young children find virtual reality systems easy to use for learning regardless of prior experience. Interestingly, the results did not confirm $\mathrm{H}_{1}$, as although subjects reported higher levels of immersion with the Google Cardboard and Samsung Gear VR than with the screen and Xbox Controller (see Fig 7), the ANOVA test did not find a statistically-significant difference in the means. One possible explanation for the similar levels of immersion provided is the nature of the exploration facilitated by the systems. The headsetbased systems facilitated exploration of the Cathedral from pre-defined points of interest hence the subjects were confined to interacting with contents from distinct viewpoints, whereas the screen-based system provided more freedom to explore the grounds beneath (by walking around) and skies above (by flying over) the cathedral. Ideally, the 3D model of the Cathedral should have been deployed to the mobile headset-based systems for exploration, but this posed a technical challenge owing to the insufficient memory and processing power of the mobile phones used in the headset-based systems as compared to the PC used in the screen-based system. That said, there is a case to be made for the use of mobile devices in heritage learning, as they enable users to perform on-site exploration of heritage sites as compared to computer systems that restrict users to indoor (off-site) usage due to their size and power requirements. An informal expedition in which university students took part in an on-site tour of the Cathedral using the Google Cardboard yielded positive feedback, and this use case will be further explored in a future study. It is arguable that if the mobile headsets were equipped with the full 3D model of the Cathedral and the ability to explore the space in a continuous fashion, subjects would have reported significantly-higher levels of immersion in the virtual environment.

Acknowledgments. Special thanks to Madras College, Fife, UK.

\section{References}

1. Kennedy, S., Fawcett, R., Miller, A., Dow, L., Sweetman, R., Field, A., Campbell, A., Oliver, I., McCaffery, J., and Allison, C. "Exploring canons \& cathedrals with Open Virtual Worlds: The recreation of St Andrews Cathedral, St Andrews day, 1318" 2013 Digit. Herit. Int. Congr., pp. 273-280, Oct. 2013. 
2. Fabola, A. Miller and Fawcett, R. "Exploring the past with Google Cardboard" 2015 Digital Heritage International Congress. 2015.

3. Milgram, P., Takemura, H., Utsumi, A., and Kishino, F. "Augmented Reality: A class of displays on the reality-virtuality continuum" Syst. Res., vol. 2351, no. Telemanipulator and Telepresence Technologies, pp. 282-292, 1994.

4. Bricken, M. "Virtual reality learning environments: potentials and challenges," ACM SIGGRAPH Comput. Graph., vol. 25, no. 3, pp. 178-184, 1991.

5. Mikropoulos, T. A., and Natsis, A. "Educational virtual environments: A ten-year review of empirical research (1999-2009)," Comput. Educ., vol. 56, no. 3, pp. 769 780, 2011.

6. Pan, Z. et al. "Virtual reality and mixed reality for virtual learning environments," Comput. Graph., vol. 30, no. 1, pp. 20-28, 2006.

7. Jacobson, J. "Ancient architecture in virtual reality; does visual immersion really aid learning?" Ph.D. dissertation, University of Pittsburgh, 2008.

8. McCaffery, J., Miller, A., and Allison, C. "Extending the use of virtual worlds as an educational platform - network island: An advanced learning environment for teaching internet routing algorithms" in CSEDU (1), pp. 279-284, 2011.

9. Perera, I. et al. "Towards successful 3D virtual learning - a case study on teaching human computer interaction" in International Conference for Internet Technology and Secured Transaction (ICITST), pp. 1-6, 2009.

10. Monahan, T., McArdle, G., and Bertolotto, M. "Virtual reality for collaborative e-learning," Comput. Educ., vol. 50, no. 4, pp. 1339-1353, 2008.

11. Davies, C., Miller, A., and Allison, C. "Mobile Cross Reality for cultural heritage" in Proc. Digit. 2013 - Fed. 19th Intl VSMM, 10th Eurographics GCH, 2nd UNESCO Mem. World Conf. Plus Spec. Sess. From CAA, Arqueol. 2.0 al., vol. 1, pp. 331-338, 2013.

12. Haugstvedt, A. C., and Krogstie, J. "Mobile augmented reality for cultural heritage: A technology acceptance study" in IEEE International Symposium on Mixed and Augmented Reality (ISMAR), pp. 247-255, 2012.

13. Coenen, T., Mostmans, L., and Naessens, K. "MuseUs: Case Study of a Pervasive Cultural Heritage Serious Game" in Journal of Computing and Cultural Heritage (JOCCH), pp. 8:1-8:19, 2013.

14. Getchell, K., Oliver, I., Miller, A., and Allison, C. "Metaverses as a platform for game based learning" Proc. - Int. Conf. Adv. Inf. Netw. Appl. AINA, pp. 1195-1202, 2010 .

15. Getchell, K., Miller, A., Nicoll, J. R., Sweetman, R. J., and Allison, C. "Games methodologies and immersive environments for virtual fieldwork" IEEE Trans. Learn. Technol., vol. 3, no. 4, pp. 281-293, 2010.

16. Huang, H. M., Rauch, U., and Liaw, S. S. "Investigating learners' attitudes toward virtual reality learning environments: Based on a constructivist approach," Comput. Educ., vol. 55, no. 3, pp. 1171-1182, 2010.

17. Suh, Y., Shin, C., and Woo, W. "A mobile phone guide: Spatial, personal, and social experience for cultural heritage" in IEEE Transactions on Consumer Electronics, pp. 2356-2364, 2009.

18. Google. Google Cardboard [Online]. Available: https://www.google.com/get/ cardboard/. [Accessed: 10 May 2016].

19. Samsung. Gear VR [Online]. Available: http://www.samsung.com/global/ galaxy/wearables/gear-vr/. [Accessed: 10 May 2016].

20. Unity3D [Online]. Available: https://unity3d.com/. [Accessed: 10 May 2016].

21. Unreal Engine [Online]. Available: https://www.unrealengine.com/. [Accessed: 10 May 2016]. 